\title{
Qualidade, compostos fenólicos e atividade antioxidante de amoras-pretas 'Tupy' e 'Guarani' armazenadas a diferentes temperaturas
}

\author{
Cristina Soethe ${ }^{(1)}$, Cristiano André Steffens ${ }^{(1)}$, Cassandro Vidal Talamini do Amarante ${ }^{(1)}$, \\ Mariuccia Schlichting De Martin ${ }^{(2)}$ e Anderson José Bortolini(1)
}

\begin{abstract}
(1)Universidade do Estado de Santa Catarina,Avenida Luiz de Camões, no2.090, CEP88520-000 Lages, SC, Brasil. E-mail: crisoethe@gmail.com, cristiano.steffens@udesc.br, cassandro.amarante@udesc.br, andersonjosebortolini@hotmail.com (2)Empresa de Pesquisa Agropecuária e Extensão Rural de Santa Catarina, Rua Abilio Franco, o 1.500, CEP 89500-000 Caçador, SC, Brasil. E-mail: mariucciamartin@epagri.sc.gov.br
\end{abstract}

Resumo - O objetivo deste trabalho foi avaliar o efeito da temperatura de armazenamento sobre a manutenção da qualidade, o teor de compostos fenólicos e a atividade antioxidante de amoras-pretas (Rubus sp.) 'Tupy' e 'Guarani'. Os frutos foram armazenados a $0,5,10$ e $15^{\circ} \mathrm{C}$. Após cinco dias de armazenamento, as amoras-pretas foram avaliadas quanto aos seguintes parâmetros: cor da epiderme, taxa respiratória, perda de massa, força para compressão, acidez titulável (AT), sólidos solúveis (SS), relação SS/AT, podridões, compostos fenólicos totais (CFT) e atividade antioxidante (AAT). O aumento da temperatura de armazenamento causou, em ambas as cultivares, o aumento da taxa respiratória, da perda de massa e da incidência de podridões, bem como redução dos teores de SS e AT, especialmente a partir de $5^{\circ} \mathrm{C}$. Não houve efeito da temperatura sobre a coloração em 'Tupy'. Em amoras 'Guarani', houve redução de croma e de ângulo hue, com o aumento da temperatura. A força para compressão, em ambas as cultivares, aumentou entre as temperaturas de 0 e $5^{\circ} \mathrm{C}$. CFT e AAT aumentaram com o incremento da temperatura em ambas as cultivares. $\mathrm{O}$ armazenamento de frutos de ambas as cultivares a $5^{\circ} \mathrm{C}$ proporciona frutos mais firmes e maior AT, além de permitir o incremento de CFT e AAT, sem comprometer o sabor e aspecto visual do fruto.

Termos para indexação: Rubus, alimento funcional, conservação, pequenos frutos.

\section{Quality, phenolic compounds, and antioxidant activity of 'Tupy' and 'Guarani' blackberries stored at different temperatures}

\begin{abstract}
The objective of this work was to evaluate the effect of storage temperature on the quality maintenance, content of phenolic compounds, and antioxidant activity of 'Tupy' and 'Guarani' blackberry (Rubus sp.) fruit. Fruit were stored at $0,5,10$, and $15^{\circ} \mathrm{C}$. After five days of storage, fruit were evaluated for the following parameters: skin color, respiratory rate, weight loss, force to compression, titratable acidity (TA), soluble solids content (SSC), SSC/TA ratio, incidence of decay, total phenolic compounds (TPC), and total antioxidant activity (TAA). In both cultivars, the temperature increase caused boosting of respiratory rate, weight loss, and incidence of decay, as well as reduction in SSC and TA, especially at temperatures over $5^{\circ} \mathrm{C}$. There was no effect of temperature on skin color of 'Tupy'. In 'Guarani' blackberries, chroma and hue angle decreased with temperature. Fruit force to compression, in both cultivars, augmented by temperature increasing between 0 and $5^{\circ} \mathrm{C}$. Values of TPC and TAA augmented with increasing of temperature in both cultivars. Fruit storage at $5^{\circ} \mathrm{C}$ for both cultivars yields firmer fruit and higher TA, while allowing the increase of TPC and TAA without impairing fruit taste and visual appearance.
\end{abstract}

Terms index: Rubus, functional food, conservation, small fruit.

\section{Introdução}

A cultura da amora-preta (Rubus sp.) encontra-se em expansão no Brasil, onde é produzida principalmente nas regiões Sul e Sudeste, com destaque para os estados do Rio Grande do Sul, Santa Catarina, Paraná, Minas Gerais, São Paulo e Espírito Santo. No ano de 2005, a área plantada com amora-preta no Brasil era de 250 ha que, nos últimos anos, aumentou cerca de $100 \%$ e chegou a aproximadamente 500 ha (Strik et al., 2007; Antunes et al., 2014). De acordo com Ilha (2012), fatores como aumento do poder aquisitivo da população e mudanças do hábito alimentar contribuíram para o aumento da produção e do consumo destes frutos nos últimos anos.

A amora-preta in natura apresenta alta qualidade nutricional, contém $85 \%$ de água e $10 \%$ de carboidratos 
(Jacques \& Zambiazi, 2011), bem como vitaminas, minerais e fibras (Guedes et al., 2013), além de fitoquímicos que apresentam propriedades benéficas à saúde, como os antioxidantes, com destaque aos compostos fenólicos (Ali et al., 2011). Estudos epidemiológicos indicam que o consumo frequente de alimentos que contenham em sua composição antioxidantes naturais está associado a baixo risco de doenças cardiovasculares e de diversos tipos de câncer (Haida et al., 2014).

A eficiência de um antioxidante é dependente de sua capacidade de sequestrar os radicais livres (Sharma \& Singh, 2013). Os compostos fenólicos são responsáveis pela transferência de hidrogênio que neutraliza a ação desses radicais (Brewer, 2011). Esses compostos são oriundos do metabolismo secundário, cuja síntese pode ser aumentada em resposta a uma condição de estresse. São essenciais para o crescimento e reprodução e atuam como agentes protetores contra patógenos (Jacques \& Zambiazi, 2011). Em alimentos, os compostos fenólicos podem contribuir para o amargor, a adstringência, a cor, o sabor, o odor e a estabilidade oxidativa (Castañeda-Ovando et al., 2009). Segundo Melo et al. (2008), a composição dos compostos fenólicos pode variar em razão da espécie, cultivar, estádio de maturação, condições climáticas e até mesmo das condições de armazenamento dos frutos.

O período de conservação pós-colheita da amora-preta é relativamente curto, em consequência da elevada taxa respiratória e da fragilidade dos frutos, o que a torna suscetível a danos mecânicos durante a colheita e o manuseio (Schaker \& Antoniolli, 2009). Segundo Cia et al. (2007), o armazenamento à temperatura ambiente resulta em elevada perda de massa e incidência de podridões, em consequência da elevada perecibilidade das amoras. Todavia, o emprego de métodos de conservação pós-colheita, como o uso do armazenamento refrigerado, pode aumentar o período de conservação desses frutos. A temperatura ideal de armazenamento para amoras-pretas situa-se entre 0 e $5^{\circ} \mathrm{C}$ (Wang \& Wang, 2009); a $0^{\circ} \mathrm{C}$, os frutos podem ser conservados durante dois ou três dias, sem que ocorra perda da qualidade (Joo et al., 2011). Contudo, frutos das cultivares 'Brazos' e 'Comanche' podem ser armazenados à temperatura de $2^{\circ} \mathrm{C}$, com qualidade por até nove dias (Antunes, 2002).
Durante a conservação pós-colheita de frutos, várias modificações químicas podem ocorrer, e a velocidade dessas modificações depende da intensidade da redução do metabolismo dos frutos (Antunes et al., 2006). Os componentes químicos dos frutos podem ser influenciados por vários fatores externos, inclusive a temperatura de armazenamento (Haida et al., 2014). Diversos estudos revelam que morangos armazenados a temperaturas mais elevadas apresentam maiores concentrações de compostos fenólicos totais e maior atividade antioxidante total, em comparação àqueles armazenados a temperaturas mais baixas (Shin et al., 2007; Shin et al., 2008). Wang \& Wang (2009) também observaram que o conteúdo de compostos fenólicos totais e a atividade antioxidante total de cranberry (Vaccinium macrocarpon) aumentou com o incremento da temperatura de armazenamento.

Atualmente, a cultivar de amora-preta 'Tupy' é a mais importante no Brasil, graças à elevada produtividade, boa capacidade de adaptação a diferentes condições edafoclimáticas e elevada qualidade dos frutos, que são bem aceitos no mercado in natura por seu tamanho, uniformidade, firmeza, coloração intensa e sabor. A cultivar 'Guarani' apresenta características similares à 'Tupy', apesar de ter uma menor área cultivada no país (Antunes et al., 2014). Contudo, até o momento, se desconhece a temperatura ideal de armazenamento, bem como sua influência sobre a qualidade e as características funcionais de ambas as cultivares.

O objetivo deste trabalho foi avaliar o efeito da temperatura de armazenamento sobre a manutenção da qualidade, o teor de compostos fenólicos e a atividade antioxidante de amoras-pretas 'Tupy' e 'Guarani'.

\section{Material e Métodos}

O experimento foi realizado em 2014, com amoras-pretas 'Tupy' e 'Guarani', provenientes de um pomar experimental localizado no Município de Lages, SC $\left(27^{\circ} 48^{\prime} 58^{\prime \prime} \mathrm{S}, 50^{\circ} 19^{\prime} 34^{\prime \prime} \mathrm{W}\right.$, a $884 \mathrm{~m}$ de altitude). As amoras foram colhidas no estádio maduro, pela manhã, acondicionadas em bandejas de polietileno tereftalato (20 frutos por bandeja) sem tampa, envoltas em policloreto de vinila $(20 \mu \mathrm{m}$ de espessura), e armazenadas durante cinco dias a 0,5 , 10 e $15^{\circ} \mathrm{C}$.

Após o armazenamento, os frutos foram avaliados quanto à cor da epiderme, taxa respiratória, perda de

Pesq. agropec. bras., Brasília, v.51, n.8, p.950-957, ago. 2016 DOI: 10.1590/S0100-204X2016000800007 
massa, força para compressão (textura), acidez titulável (AT), sólidos solúveis (SS), relação SS/AT, incidência de podridões, teor de compostos fenólicos totais (CFT) e atividade antioxidante total (AAT, medida pelos métodos DPPH e ABTS).

A taxa respiratória $\left(\mu \mathrm{mol} \mathrm{CO} \mathrm{Kg}^{-1} \mathrm{~s}^{-1}\right)$ foi quantificada em frutos de cada amostra colocados em um recipiente com $480 \mathrm{~mL}$, hermeticamente fechado. Após $60 \mathrm{~min}$, amostrou-se a atmosfera interna do recipiente por meio de seringas de $1 \mathrm{~mL}$ de volume, e quantificaram-se as concentrações de $\mathrm{CO}_{2}$, em um cromatógrafo CP-3800 (Varian, Palo Alto, CA, EUA), equipado com coluna porapak $\mathrm{N}$ de $3 \mathrm{~m}$ de comprimento, metanador $\mathrm{e}$ detector de ionização de chama.

A cor da epiderme foi avaliada quanto à luminosidade (L), croma (C) e ângulo hue $\left(\mathrm{h}^{\mathrm{o}}\right)$, com o auxílio de um colorímetro CR 400 (Konica Minolta, Tóquio, Japão). As leituras foram realizadas em dois pontos opostos da região equatorial dos frutos. Os valores de $\mathrm{h}^{\mathrm{o}}$ apresentaram as seguintes correspondências quanto às cores da superfície do tecido vegetal: $0 \%$ vermelho, $90 \%$ amarelo, $180^{\circ} /$ verde e $270 \%$ azul. Os valores de $\mathrm{L}$ variaram de 0 (preto) a 100 (branco). Assim, quanto menor os valores de L, mais escurecidos estariam os frutos.

A força necessária para a compressão dos frutos (textura) foi analisada com texturômetro eletrônico TAXT-Plus (Stable Micro Systems Ltd, Surrey, Reino Unido). Utilizou-se uma plataforma plana, modelo $\mathrm{P} / 75$, com $75 \mathrm{~mm}$ de diâmetro, que exerceu uma força de compressão até a deformação de $3 \mathrm{~mm}$ na superfície do fruto.

Os valores de AT (\% ácido cítrico) foram obtidos em uma amostra de $10 \mathrm{~mL}$ de suco, obtido pelo processamento dos frutos em uma centrífuga. Essa amostra foi diluída em $90 \mathrm{~mL}$ de água destilada e titulada com solução de $\mathrm{NaOH} 0,1 \mathrm{~N}$ até $\mathrm{pH} 8,1$. Para a titulação, utilizou-se titulador automático TitroLine Easy (Schott Instruments, Mainz, Rheinland-Pfalz, Alemanha). Os teores de SS (\%) foram determinados em um refratômetro digital PR201 $\alpha$ (Atago, Tóquio, Japão), em uma alíquota do suco obtido pelo processamento dos frutos.

A incidência de podridões (\%) foi avaliada pela contagem dos frutos afetados com características de infecção por patógenos. A perda de massa (\%) foi avaliada pela diferença de massa entre a colheita e a saída da câmara.
Para a obtenção dos extratos de amora, quantificaram-se os compostos fenólicos totais (CFT) e a atividade antioxidante total (AAT), em $5 \mathrm{~g}$ de amostra, que foi homogeneizada com $10 \mathrm{~mL}$ de etanol acidificado $(0,01 \%$ de $\mathrm{HCl})$ e centrifugada a $15.000 \mathrm{rpm}$, a $4^{\circ} \mathrm{C}$ por $5 \mathrm{~min}$. Após a filtragem, o sobrenadante foi reservado para a análise de CFT e AAT.

A determinação de CFT foi realizada com o reagente de Folin-Ciocalteu. A curva-padrão foi obtida com ácido gálico, às concentrações de $0,10,30,50,70,90$ e $100 \mathrm{ppm}$. Para a análise adicionaram-se $2,5 \mathrm{~mL}$ de Folin-Ciocalteu (1:3), 0,5 mL de amostra e 2,0 mL de solução de carbonato de sódio a $10 \%$. Os tubos foram agitados e incubados por uma hora ao abrigo da luz. Realizou-se a leitura do comprimento de onda $(\lambda)$ de $765 \mathrm{~nm}$, em espectrofotômetro UV-visível, modelo U-1100 (Hitachi, Tóquio, Japão). Os resultados foram expressos em miligramas de equivalentes de ácido gálico por $100 \mathrm{~g}$ de massa de amostra fresca $(\mathrm{mg}$ EAG $\left.100 \mathrm{~g}^{-1}\right)$.

A determinação da AAT foi baseada na extinção da absorçãodosradicaisDPPH(2,2-difenil-1-picril-hidrazil) e ABTS (2,2'-Azino-bis(3-etilbenzotiazolin-6-ácido sulfônico). O método DPPH foi analisado de acordo com Vizzotto et al. (2012). Em ambiente escuro, $200 \mu \mathrm{L}$ de amostra foram pipetados e misturados com $3.800 \mu \mathrm{L}$ de radical DPPH. Os tubos foram agitados e deixados para reagir por 24 horas. A leitura foi realizada em $\lambda=525 \mathrm{~nm}$, e os resultados foram expressos em microgramas de equivalente Trolox $\mathrm{g}^{-1}$ de massa de amostra fresca. $\mathrm{O}$ método ABTS foi analisado conforme descrito por Rufino et al. (2007) com adaptações. Em ambiente escuro, $30 \mu \mathrm{L}$ de amostra foram pipetados e misturados com $3.000 \mu \mathrm{L}$ de radical ABTS. A leitura foi realizada após reação de $6 \min$ em $\lambda=734 \mathrm{~nm}$, e os resultados foram expressos em microgramas de equivalente Trolox $\mathrm{g}^{-1}$ de massa de amostra fresca.

Utilizou-se o delineamento inteiramente casualizado com quatro repetições, e a unidade experimental constituída de 20 frutos. Os dados foram submetidos à análise estatística com o auxílio do programa SAS (SAS Institute Inc.). O efeito da temperatura sobre os atributos avaliados foi analisado por meio de contrastes ortogonais polinomiais (lineares e quadráticos). 


\section{Resultados e Discussão}

À colheita, as amoras 'Tupy' apresentaram os seguintes valores: SS, 8,63\%; AT, 1,40\% de ácido cítrico; relação SS/AT, 6,25; L, 13,3; C, 2,08; ho , 73,2; força para compressão, 4,34 N; CFT, 229,7 mg EAG $100 \mathrm{~g}^{-1}$; AAT, pelo método DPPH, 9.373,5 $\mu \mathrm{g}$ de eq. Trolox $\mathrm{g}^{-1}$, e ATT pelo método ABTS, 32,2 $\mu \mathrm{g}$ de eq. Trolox $\mathrm{g}^{-1}$. Amoras 'Guarani' apresentaram, à colheita: SS, 8,45\%; AT, $1,45 \%$ de ácido cítrico; relação SS/AT, 5,87; L, 14,5; C, 2,17; ho , 67,6; força para compressão, 4,65 N; CFT, 253,4 mg EAG $100 \mathrm{~g}^{-1}$; AAT, pelo método DPPH, 10.714,5 $\mu \mathrm{g}$ de eq. Trolox $\mathrm{g}^{-1}$, e ATT pelo método ABTS, 30,6 $\mu \mathrm{g}$ de eq. Trolox $\mathrm{g}^{-1}$.

Independentemente da cultivar utilizada, houve menor taxa respiratória nos frutos armazenados a temperaturas mais baixas do que naqueles armazenados a temperaturas mais elevadas (Tabela 1). Em amoras 'Tupy', a redução da respiração com a diminuição da temperatura de armazenamento apresentou comportamento linear. Em amoras 'Guarani', a respiração apresentou um comportamento quadrático, tendo-se observado pequena redução entre as temperaturas de 15 a $5^{\circ} \mathrm{C}$. Geisen et al. (2005) também observaram redução da taxa respiratória com a redução da temperatura de armazenamento em morangos. $\mathrm{O}$ armazenamento a baixas temperaturas resulta em redução da atividade respiratória de frutos e, consequentemente, conduz a um aumento do período de conservação (Antunes et al., 2003).

Tabela 1. Taxa respiratória, perda de massa de matéria fresca e incidência de podridões em amoras-pretas (Rubus sp.) 'Tupy' e 'Guarani', armazenadas durante cinco dias a diferentes temperaturas.

\begin{tabular}{|c|c|c|c|c|c|c|}
\hline \multirow[t]{2}{*}{$\begin{array}{l}\text { Temperatura } \\
\left({ }^{\circ} \mathrm{C}\right)\end{array}$} & \multicolumn{2}{|c|}{$\begin{array}{c}\text { Taxa respiratória } \\
\left(\mu \mathrm{mol} \mathrm{CO}_{2} \mathrm{~kg}^{-1} \mathrm{~s}^{-1}\right)\end{array}$} & \multicolumn{2}{|c|}{$\begin{array}{l}\text { Perda de massa de } \\
\text { matéria fresca }(\%)\end{array}$} & \multicolumn{2}{|c|}{$\begin{array}{l}\text { Incidência de } \\
\text { podridões }(\%)\end{array}$} \\
\hline & 'Tupy' & 'Guarani' & 'Tupy' & 'Guarani' & 'Tupy' & 'Guarani' \\
\hline 0 & 0,69 & 1,37 & 0,76 & 0,61 & 10,67 & 0,91 \\
\hline 5 & 0,88 & 2,19 & 1,29 & 0,97 & 26,25 & 28,43 \\
\hline 10 & 0,89 & 2,26 & 2,35 & 1,75 & 50,00 & 37,17 \\
\hline 15 & 1,13 & 2,36 & 3,07 & 2,19 & 81,82 & 50,00 \\
\hline Linear $^{(1)}$ & $* * *$ & $* * *$ & $* * *$ & $* * *$ & $* * *$ & $* * *$ \\
\hline Quadrático $^{(1)}$ & ns & $* *$ & $* *$ & ns & ns & * \\
\hline CV (\%) & 12,68 & 9,77 & 6,42 & 12 & 30,27 & 44,17 \\
\hline
\end{tabular}

${ }^{(1)}$ Efeitos linear e quadrático da temperatura de armazenamento, analisados por meio de contrastes ortogonais polinomiais. ns Não significativo. *, **, $* * *$ Significativo a 5,1 e $0,1 \%$ de probabilidade, respectivamente.
Houve menor perda de massa de matéria fresca e menor incidência de podridões, em ambas as cultivares, nos frutos armazenados a temperaturas mais baixas do que nos armazenados a temperaturas mais elevadas (Tabela 1). A perda de massa de matéria fresca em frutos armazenados resulta principalmente da transpiração (Joo et al., 2011). Todavia, a menor perda de massa, em resposta à redução da temperatura de armazenamento, decorre principalmente da diminuição do deficit de pressão de vapor entre o fruto e o ar da atmosfera do ambiente de armazenamento. Embora a perda de massa, no presente trabalho, tenha aumentado com o incremento da temperatura, os valores observados, mesmo a temperaturas mais altas, não foram muito elevados e atingiram os máximos de 3,1 e 2,2\%, nas amoras 'Tupy' e 'Guarani', respectivamente, sem causar depreciação visual à qualidade dos frutos pelo murchamento. A baixa perda de massa de matéria fresca observada pode estar relacionada à alta umidade no interior da embalagem, uma vez que os frutos foram acondicionados em bandejas de polietileno tereftalato e envoltas com filme de policloreto de vinila com espessura de $20 \mu \mathrm{m}$. A alta umidade relativa no interior da embalagem é desejável, pois reduz o deficit de pressão de vapor, contudo, pode aumentar a incidência de podridões (Shin et al., 2007). A elevada incidência de podridões, especialmente em amoras 'Tupy', entre $10 \mathrm{e}$ $15^{\circ} \mathrm{C}$, pode estar relacionada à combinação de fatores, como temperatura elevada, alta umidade e insuficiente modificação da atmosfera no interior da embalagem (Cia et al., 2007). No entanto, amoras 'Guarani' à $0^{\circ} \mathrm{C}$ apresentaram baixa incidência de podridão $(<1 \%)$, e, nessa condição, os frutos podem ser armazenados por um período de cinco dias.

Houve redução dos teores de SS e de AT com o aumento da temperatura de armazenamento, com redução mais expressiva a partir de $10^{\circ} \mathrm{C}$ para $\mathrm{AT}$, e a partir de $15^{\circ} \mathrm{C}$ para SS. Contudo, não houve efeito da temperatura de armazenamento para a relação SS/ AT, em ambas as cultivares (Tabela 2). Os menores valores de AT e de SS, a temperaturas mais elevadas de armazenamento, decorrem da maior atividade respiratória nos frutos (Tabela 1). Com o aumento da temperatura de armazenamento, os processos enzimáticos degradativos tornam-se favoráveis, o que resulta em consumo de açúcares e de ácidos orgânicos utilizados como substratos respiratórios (Antunes et al., 2003). Ayala-Zavala et al. (2004), em pesquisa sobre 
morangos, também observaram menor teor de SS em frutos armazenados a $10^{\circ} \mathrm{C}$ do que a temperaturas de 0 e $5^{\circ} \mathrm{C}$. No entanto, Ayala-Zavala et al. (2004) e Shin et al. (2007) não observaram efeito da temperatura de armazenamento sobre a AT em morangos.

Em amoras 'Tupy', não se observaram diferenças entre as temperaturas de armazenamento para os atributos de cor da epiderme. Em amoras 'Guarani', houve aumento dos valores de $\mathrm{L}$ até a temperatura de $10{ }^{\circ} \mathrm{C}$, com posterior redução. Os valores de $\mathrm{C}$ apresentaram redução com o incremento da temperatura. Os valores de $\mathrm{h}^{\circ}$ reduziram-se com o aumento da temperatura de armazenamento, com pequena redução entre as temperaturas de 10 e $15^{\circ} \mathrm{C}$ (Tabela 3). As temperaturas de armazenamento de $0 \mathrm{e}$ de $15^{\circ} \mathrm{C}$ proporcionaram menores valores de $\mathrm{L}$, o que nos indica que amoras 'Guarani', armazenadas a essas

Tabela 2. Sólidos solúveis (SS), acidez titulável (AT) e relação SS/AT de amoras-pretas (Rubus sp.) 'Tupy' e 'Guarani', armazenadas durante cinco dias a diferentes temperaturas.

\begin{tabular}{|c|c|c|c|c|c|c|}
\hline \multirow{2}{*}{$\begin{array}{l}\text { Temperatura } \\
\left({ }^{\circ} \mathrm{C}\right)\end{array}$} & \multicolumn{2}{|c|}{$\mathrm{SS}(\%)$} & \multicolumn{2}{|c|}{ AT $(\%)$} & \multicolumn{2}{|c|}{$\mathrm{SS} / \mathrm{AT}$} \\
\hline & 'Tupy' & 'Guarani' & 'Tupy' & 'Guarani' & 'Tupy' & 'Guarani' \\
\hline 0 & 8,33 & 8,43 & 1,51 & 1,25 & 5,52 & 6,74 \\
\hline 5 & 7,93 & 8,35 & 1,51 & 1,22 & 5,25 & 6,84 \\
\hline 10 & 7,58 & 8,23 & 1,25 & 1,06 & 6,06 & 7,76 \\
\hline 15 & 5,73 & 7,05 & 1,17 & 1,00 & 4,90 & 7,05 \\
\hline Linear $^{(1)}$ & $* * *$ & $* * *$ & $*$ & $* *$ & ns & ns \\
\hline Quadrático $^{(1)}$ & $*$ & $*$ & $\mathrm{~ns}$ & ns & ns & ns \\
\hline$\overline{\mathrm{CV}(\%)}$ & 6,89 & 3,8 & 17,88 & 11,84 & 22,27 & 13,24 \\
\hline
\end{tabular}

${ }^{(1)}$ Efeitos linear e quadrático de temperatura de armazenamento, analisados por meio de contrastes ortogonais polinomiais. ns Não significativo. *, **, ***Significativo a 5,1 e $0,1 \%$ de probabilidade, respectivamente. temperaturas, têm coloração mais escura. Shin et al. (2007) observaram, em morangos, menores valores de $\mathrm{L}$ em frutos armazenados durante quatro dias a $20^{\circ} \mathrm{C}$, do que naqueles armazenados a 10 e $0,5^{\circ} \mathrm{C}$.

Os valores de $\mathrm{C}$ expressam a intensidade da cor, ou seja, a saturação em termos de pigmentos da cor, assumindo-se valores próximos à zero para cores neutras (cinza) e próximos de 60 para cores vívidas (Silva et al., 2011). Para amoras 'Guarani', os frutos armazenados em temperaturas mais baixas obtiveram valor de $\mathrm{C}$ maior do que naqueles armazenados a temperaturas mais elevadas (Tabela 3), o que mostra que os frutos armazenados nessas condições apresentam maior intensidade de cor do que os frutos armazenados a temperaturas mais altas. Shin et al. (2008) também observaram maior valor de $\mathrm{C}$ em morango, após 12 dias de armazenamento a temperaturas mais baixas, do que naqueles armazenados a temperaturas mais elevadas. A intensidade da cor é um fator importante, tendo-se em vista que a coloração é um dos atributos de qualidade de relevância para o consumidor.

Em amoras 'Guarani', observou-se que temperaturas de armazenamento mais altas proporcionaram os menores valores de $h^{\circ}$ do que em frutos armazenados a temperaturas mais baixas (Tabela 3 ). Todavia, a maior redução de $h^{\circ}$ foi até a temperatura de $10^{\circ} \mathrm{C}$, com pouca alteração em relação aos frutos armazenados a $15^{\circ} \mathrm{C}$. Nota-se ainda, que não houve diferença de valores de $\mathrm{h}^{\circ}$ nos frutos armazenados a $0^{\circ} \mathrm{C}$, em comparação à data da colheita. Isso indica que, para amoras 'Guarani', a temperatura de $0^{\circ} \mathrm{C}$ mantém a cor dos frutos. Shin et al. (2008) também observaram maior redução do valor de $\mathrm{h}^{\circ}$ em morangos armazenados a $10^{\circ} \mathrm{C}$ do que nos frutos armazenados a $3^{\circ} \mathrm{C}$, após nove

Tabela 3. Atributos de cor (luminosidade, L; croma, C; e ângulo hue, $\mathrm{h}^{\circ}$ ) e força para compressão de amoras-pretas (Rubus sp.) 'Tupy' e 'Guarani', armazenadas durante cinco dias a diferentes temperaturas.

\begin{tabular}{|c|c|c|c|c|c|c|c|c|}
\hline \multirow{2}{*}{$\begin{array}{l}\text { Temperatura } \\
\left({ }^{\circ} \mathrm{C}\right)\end{array}$} & \multicolumn{2}{|c|}{ Luminosidade } & \multicolumn{2}{|c|}{ Croma } & \multicolumn{2}{|c|}{ Ângulo hue $\left(\mathrm{h}^{\mathrm{o}}\right)$} & \multicolumn{2}{|c|}{ Força para compressão $(\mathrm{N})$} \\
\hline & 'Tupy' & 'Guarani' & 'Tupy' & 'Guarani' & 'Tupy' & 'Guarani' & 'Tupy' & 'Guarani' \\
\hline 0 & 15,11 & 13,66 & 2,31 & 2,16 & 63,80 & 67,66 & 3,41 & 3,87 \\
\hline 5 & 15,62 & 15,35 & 2,24 & 2,04 & 60,85 & 62,62 & 3,71 & 4,4 \\
\hline 10 & 16,06 & 15,91 & 2,22 & 1,86 & 58,93 & 59,65 & 2,67 & 2,92 \\
\hline 15 & 14,81 & 13,9 & 2,43 & 1,90 & 59,17 & 59,53 & 1,63 & 2,26 \\
\hline Linear $^{(1)}$ & ns & ns & ns & $*$ & ns & $* *$ & $* * *$ & $* * *$ \\
\hline Quadrático $^{(1)}$ & ns & $* * *$ & ns & ns & $\mathrm{ns}$ & $*$ & $*$ & $* *$ \\
\hline $\mathrm{CV}(\%)$ & 6,04 & 5,2 & 17,2 & 8,51 & 6,11 & 4,57 & 16,32 & 8,3 \\
\hline
\end{tabular}

${ }^{(1)}$ Efeitos linear e quadrático de temperatura de armazenamento, analisados por meio de contrastes ortogonais polinomiais. ${ }^{\text {ns Não significativo. } *, * *, * * * \text { Sig- }}$ nificativo a 5,1 e $0,1 \%$ de probabilidade, respectivamente. 
dias de armazenamento. De acordo com Ayala-Zavala et al. (2004), temperaturas de armazenamento mais altas resultam em aumento do processo de maturação dos frutos, que pode ocasionar redução dos valores de $\mathrm{h}^{\circ}$. Embora alterações significativas tenham ocorrido nos atributos de cor em amoras 'Guarani', em razão da temperatura de armazenamento, as diferenças entre os valores observados foram pequenas, inclusive em relação aos valores obtidos na colheita, o que mostra que a alteração da cor das amoras foi pequena durante o armazenamento. Cabe salientar que, ao olho humano, as diferenças encontradas não são perceptíveis.

A força para compressão do fruto, em ambas as cultivares, aumentou entre as temperaturas de 0 e $5^{\circ} \mathrm{C}$, com posterior redução, com o aumento da temperatura de armazenamento (Tabela 3). Durante o armazenamento dos frutos, o cálcio pode ser liberado das paredes celulares e levar à solubilização da protopectina, pela ação de enzimas que são influenciadas pela temperatura de armazenamento, o que causa a redução da firmeza dos frutos (Antunes et al., 2006). Além disso, a redução da firmeza dos frutos, durante o armazenamento, também pode ocorrer em consequência da diminuição da turgescência das células, decorrente do armazenamento dos frutos em condições de baixa umidade relativa do ar, que propicia a perda de água e reduz a resistência do fruto à deformação. Todavia, o incremento da firmeza de polpa, com o aumento da temperatura entre 0 e $5^{\circ} \mathrm{C}$, pode ocorrer graças ao aumento da viscosidade da pectina (Shin et al., 2007).

O teor de CFT aumentou com o incremento da temperatura de armazenamento, nas duas cultivares. Em amoras 'Guarani', o maior aumento do teor de CFT foi observado entre as temperaturas de 0 e $5^{\circ} \mathrm{C}$, em comparação aos frutos armazenados nas demais temperaturas. No entanto, em amoras 'Tupy', o aumento do teor de CFT foi linear entre as temperaturas de armazenamento (Tabela 4). Ayala-Zavala et al. (2004) trabalharam com morangos, e Kivi et al. (2014), com framboesa, e também observaram aumento do teor de CFT, com o incremento da temperatura de armazenamento. A síntese de compostos fenólicos deriva da oxidação parcial de açúcares e ácidos orgânicos na glicólise e no ciclo dos ácidos tricarboxílicos. É possível que parte dos ácidos e açúcares, consumidos na respiração, tenham sido utilizados como fonte de esqueletos de carbono para a síntese de compostos fenólicos, uma vez que as temperaturas mais elevadas de armazenamento apresentaram maior redução da AT e dos SS, bem como maior taxa respiratória (Tabelas 1 e 2).

Em relação à colheita, houve redução do teor de CFT nos frutos armazenados a $0^{\circ} \mathrm{C}$, em ambas as cultivares (Tabela 4). Porém, esse efeito foi mais pronunciado em amoras 'Guarani'. A diminuição do teor de CFT durante o armazenamento pode ter ocorrido em consequência de sua oxidação, que reduziu sua concentração no fruto (Yang et al., 2011). A temperaturas mais baixas, pode haver produção de espécies reativas de oxigênios, resultando em maior consumo de compostos fenólicos (Junmatong et al., 2015). Além disso, como a síntese de compostos fenólicos deriva da oxidação parcial de açúcares e de ácidos orgânicos na glicólise e no ciclo dos ácidos tricarboxílicos, os frutos armazenados a baixas temperaturas (temperatura de $0^{\circ} \mathrm{C}$ ), apresentam maior retardo do metabolismo (maior conteúdo de $\mathrm{SS}$, AT e menor taxa respiratória) (Tabelas 1 e 2), resultando em menor síntese de compostos fenólicos (Pérez-Ilzarbe et al., 1997), em função da menor produção de esqueletos carbônicos.

A AAT das amoras, independentemente da cultivar e do método de avaliação (DPPH e ABTS), aumentou com o incremento da temperatura de armazenamento em comparação àquelas armazenadas em temperaturas mais baixas (Tabela 4). Para amoras 'Guarani', maior aumento da AAT foi observado entre as temperaturas de 0 e $5^{\circ} \mathrm{C}$, comparado com os frutos armazenados

Tabela 4. Compostos fenólicos totais (CFT) e atividade antioxidante total (AAT) de amoras-pretas (Rubus sp.) 'Tupy' e 'Guarani' - quantificada pelos métodos DPPH e ABTS -, em frutos armazenados durante cinco dias a diferentes temperaturas.

\begin{tabular}{|c|c|c|c|c|c|c|}
\hline \multirow{3}{*}{$\begin{array}{l}\text { Temperatura } \\
\left({ }^{\circ} \mathrm{C}\right)\end{array}$} & \multirow{2}{*}{\multicolumn{2}{|c|}{$\begin{array}{c}\text { CFT } \\
\left(\mathrm{mg} \mathrm{EAG} 100 \mathrm{~g}^{-1}\right)\end{array}$}} & \multicolumn{4}{|c|}{ AAT ( $\mu$ g eq. Trolox $\mathrm{g}^{-1}$ de massa de MF) } \\
\hline & & & \multicolumn{2}{|c|}{ DPPH } & \multicolumn{2}{|c|}{ ABTS } \\
\hline & 'Tupy' & 'Guarani' & 'Tupy' & 'Guarani' & 'Tupy' & 'Guarani' \\
\hline 0 & 206,4 & 123,2 & 8.535 & 5.462 & 26,4 & 11,2 \\
\hline 5 & 236,9 & 262,8 & 8.924 & 13.898 & 33,7 & 28,4 \\
\hline 10 & 254,3 & 285,8 & 10.628 & 14.050 & 33,9 & 32,1 \\
\hline 15 & 275,3 & 300,1 & 10.797 & 14.957 & 41,2 & 35,0 \\
\hline Linear $^{(1)}$ & $* * *$ & $* * *$ & ** & $* * *$ & $* * *$ & $* * *$ \\
\hline Quadrático $^{(1)}$ & ns & $* * *$ & ns & $* * *$ & ns & $* * *$ \\
\hline $\mathrm{CV}(\%)$ & 9,52 & 8,47 & 11,78 & 7,92 & 8,59 & 7,19 \\
\hline
\end{tabular}

${ }^{(1)}$ Efeitos linear e quadrático de temperatura de armazenamento, analisados por meio de contrastes ortogonais polinomiais. ns Não significativo. *, **, ***Significativo a 5,1 e $0,1 \%$ de probabilidade, respectivamente. EAG, equivalente de ácido gálico; MF, matéria fresca. 
nas demais temperaturas, quando os incrementos foram menores. Por outro lado, para amoras 'Tupy', o aumento da AAT foi linear com o incremento na temperatura. Ayala-Zavala et al. (2004) também verificaram aumento da AAT com o incremento da temperatura de armazenamento em morangos. Segundo Acosta-Montoya et al. (2010), a amora possui elevada atividade antioxidante, que pode ser explicada pelo efeito sinérgico entre os compostos fenólicos. De acordo com Rotili et al. (2013), a atividade antioxidante em frutos é decorrente da ação de uma variedade de compostos que são degradados ou sintetizados durante o armazenamento, em resposta a estresses bióticos e abióticos. No caso de amoras-preta 'Tupy' e 'Guarani', a temperatura de armazenamento de $5^{\circ} \mathrm{C}$ foi a que melhor manteve a AAT, com resultados mais próximos aos encontrados na colheita dos frutos. A temperatura de $0^{\circ} \mathrm{C}$ reduziu, enquanto as temperaturas de 10 e $15^{\circ} \mathrm{C}$ aumentaram a AAT dos frutos, em comparação aos valores encontrados na colheita. Todavia, é importante salientar que apesar de altos níveis de antioxidantes serem desejáveis e benéficos a saúde humana, temperaturas mais elevadas são prejudiciais para a manutenção da qualidade das amoras.

Apesar do armazenamento sob temperaturas mais elevadas $\left(10\right.$ e $\left.15^{\circ} \mathrm{C}\right)$ ter ocasionado um incremento nos teores de CFT e na ATT, o armazenamento sob tais condições proporcionou maior perda de massa e incidência de podridões, depreciando a aparência dos frutos. Além disso, essas condições de armazenamento reduziram os teores de $\mathrm{SS}$ e a $\mathrm{AT}$ e prejudicaram a textura dos frutos, comprometendo a palatabilidade. Assim sendo, ainda que o aumento da temperatura tenha ocasionado um efeito positivo sobre o teor de compostos fenólicos e a atividade antioxidante dos frutos, o armazenamento sob altas temperaturas se mostrou inviável, devido ao comprometimento da qualidade dos mesmos.

\section{Conclusões}

1. $\mathrm{O}$ armazenamento a $0^{\circ} \mathrm{C}$ mantém a qualidade físico-química dos frutos, porém inibe o incremento nos teores de compostos fenólicos totais e da atividade antioxidante total de amora-preta das cultivares 'Tupy' e 'Guarani'.

2. $\mathrm{O}$ armazenamento a $5^{\circ} \mathrm{C}$ proporciona maior firmeza de polpa, acidez titulável elevada e permite satisfatório incremento no teor de compostos fenólico totais e da atividade antioxidante total, sem alterar a relação sólidos solúveis/acidez titulável e causar grandes prejuízos ao aspecto visual dos frutos.

\section{Referências}

ACOSTA-MONTOYA, O.; VAILLANT, F.; COZZANO, S.; MERTZ, C.; PÉREZ, A.M.; CASTRO, M.V. Phenolic content and antioxidant capacity of tropical highland blackberry (Rubus adenotrichus Schltdl.) during three edible maturity stages. Food Chemistry, v.119, p.1497-1501, 2010. DOI: 10.1016/j. foodchem.2009.09.032.

ALI, L.; SVENSSON, B.; ALSANIUS, B.W.; OLSSON, M.E. Late season harvest and storage of Rubus berries - major antioxidant and sugar levels. Scientia Horticulturae, v.129, p.376-381, 2011. DOI: 10.1016/j.scienta.2011.03.047.

ANTUNES, L.E.C. Amora-preta: nova opção de cultivo no Brasil. Ciência Rural, v.32, p.151-158, 2002. DOI: 10.1590/ S0103-84782002000100026.

ANTUNES, L.E.C.; DUARTE FILHO, J.; SOUZA, C.M. de. Conservação pós-colheita de frutos de amoreira-preta. Pesquisa Agropecuária Brasileira, v.38, p.413-419, 2003. DOI: 10.1590/ S0100-204X2003000300011.

ANTUNES, L.E.C.; GONÇALVES, E.D.; TREVISAN, R. Alterações da atividade da poligalacturonase e pectinametilesterase em amora-preta (Rubus spp.) durante o armazenamento. Revista Brasileira de Agrociência, v.12, p.63-66, 2006.

ANTUNES, L.E.C.; PEREIRA, I. dos S.; PICOLOTTO, L.; VIGNOLO, G.K.; GONÇALVES, M.A. Produção de amoreira-preta no Brasil. Revista Brasileira de Fruticultura, v.36, p.100-111, 2014.

AYALA-ZAVALA， J.F.; WANG， S.Y.; WANG， C.Y.; GONZÁLEZ-AGUILAR, G.A. Effect of storage temperatures on antioxidant capacity and aroma compounds in strawberry fruit. Food Science and Technology, v.37, p.687-695, 2004. DOI: 10.1016/j.lwt.2004.03.002.

BREWER, M.S. Natural antioxidants: Sources, compounds, mechanisms of action, and potential applications. Comprehensive Reviews in Food Science and Food Safety, v.10, p.221-247, 2011. DOI: $10.1111 /$ j.1541-4337.2011.00156.x.

CASTAÑEDA-OVANDO, C.A.; PACHECO-HERNÁNDEZ, M.L.; PÁEZ-HERNÁNDEZ, M.E.; RODRÍGUEZ, J.A.; GALÁN-VIDAL, C.A. Chemical studies of anthocyanins: A review. Food Chemistry, v.113, p.859-871, 2009. DOI: 10.1016/j. foodchem.2008.09.001.

CIA, P.; BRON, I.U.; VALENTINI, S.R. de T.; PIO, R.; CHAGAS, E.A. Atmosfera modificada e refrigeração para conservação pós-colheita da amora-preta. Bioscience Journal, v.23, p.11-16, 2007.

GEISEN, S.; VERLINDEN, B.E.; CONESA, A.; NICOLAI, B.M. Modelling respiration of strawberry (cv. 'Elsanta') as a function of temperature, carbon dioxide, low and superatmospheric oxygen 
concentrations. Information and Technology for Sustainable Fruit and Vegetable Production, v.5, p.119-126, 2005.

GUEDES, M.N.S.; ABREU, C.M.P. de; MARO, L.A.C.; PIO, R.; ABREU, J.R. de; OLIVEIRA, J.O. de. Chemical characterization and mineral levels in the fruits of blackberry cultivars grown in a tropical climate at an elevation. Acta Scientiarum. Agronomy, v.35, p.191-196, 2013. DOI: 10.4025/ actasciagron.v35i2.16630.

HAIDA, K.S.; SILVA, F.J. da; COELHO, S.R.M.; LIMA, D.S. de; ABRÃO, R.M.; HAIDA, K.Y. Caracterização físico-química e atividade antioxidante de amoreira-preta (Morus nigra L.). Revista Brasileira de Ciências da Saúde, ano12, p.21-28, 2014. DOI: 10.13037/rbcs.vol12n40.2185.

ILHA, L.L.H. Produção de amora-preta e framboesa em regiões de clima temperado. Informe Agropecuário, v.33, p.58-68, 2012.

JACQUES, A.C.; ZAMBIAZI, R.C. Fitoquímicos em amora-preta (Rubus spp.). Semina: Ciências Agrárias, v.32, p.245-260, 2011. DOI: $10.5433 / 1679-0359.2011 v 32 n 1 p 245$.

JOO, M.; LEWANDOWSKI, N.; AURAS, R.; HARTE, J.; ALMENAR, E. Comparative shelf life study of blackberry fruit in bio-based and petroleum-based containers under retail storage conditions. Food Chemistry, v.126, p.1734-1740, 2011. DOI: 10.1016/j.foodchem.2010.12.071.

JUNMATONG, C.; FAIYUE, B.; ROTARAYANONT, S.; UTHAIBUTRA, J.; BOONYAKIAT, D.; SAENGNIL, $\mathrm{K}$. Cold storage in salicylic acid increases enzymatic and non-enzymatic antioxidants of 'Nam Dok Mai no. 4' mango fruit. ScienceAsia, v.41, p.12-21, 2015. DOI: 10.2306/ scienceasia1513-1874.2015.41.012.

KIVI, A.R.; SARTIPNIA, N.; KHALKHALI, M.B. Effect of storage temperatures on antioxidant capacity and bioactive compounds in raspberry fruit. International Journal of Plant, Animal and Environmental Sciences, v.4, p.343-349, 2014.

MELO, E. de A.; MACIEL, M.I.S.; LIMA, V.L.A.G. de; NASCIMENTO, R.J. Capacidade antioxidante de frutas. Revista Brasileira de Ciências Farmacêuticas, v.44, p.193-201, 2008. DOI: $10.1590 /$ S1516-93322008000200005.

PÉREZ-ILZARBE， J.; HERNÁNDEZ， T.; ESTRELLA， I.; VENDRELL, M. Cold storage of apples (cv. Granny Smith) and changes in phenolic compounds. Zeitschrift für Lebensmitteluntersuchung und -Forschung A, v.204, p.52-55, 1997.

ROTILI, M.C.C.; COUTRO, S.; CELANT, V.M.; VORPAGEL, J.A.; BARP, F.K.; SALIBE, A.B.; BRAGA, G.C. Composição, atividade antioxidante e qualidade do maracujá-amarelo durante armazenamento. Semina: Ciências Agrárias, v.34, p.227-240, 2013. DOI: 10.5433/1679-0359.2013v34n1p227.

RUFINO, M. do S.M.; ALVES, R.E.; BRITO, E. S. de; MORAIS, S.M. de; SAMPAIO, C. de G.; PÉREZ-JIMÉNEZ, J.; SAURA-CALIXTO, F.D. Metodologia científica: determinação da atividade antioxidante total em frutas pela captura do radical livre $\mathrm{ABTS}^{+}$. Fortaleza: Embrapa Agroindústria Tropical, 2007. 4p. (Comunicado técnico, 128).

SCHAKER, P.D.C.; ANTONIOLLI, L.R. Aspectos econômicos e tecnológicos em pós-colheita de amoras-pretas (Rubus spp.). Revista Brasileira de Agrociência, v.15, p.11-15, 2009.

SHARMA, P.; SINGH, R.P. Evaluation of antioxidant activity in foods with special reference to TEAC method. American Journal of Food Technology, v.8, p.83-101, 2013. DOI: 10.3923/ ajft.2013.83.101.

SHIN, Y.; LIU, R.H.; NOCK, J.F.; HOLLIDAY, D.; WATKINS, C.B. Temperature and relative humidity effects on quality, total ascorbic acid, phenolics and flavonoid concentrations, and antioxidant activity of strawberry. Postharvest Biology and Technology, v.45, p.349-357, 2007. DOI: 10.1016/j.postharvbio.2007.03.007.

SHIN, Y.; RYU, J.-A.; LIU, R.H.; NOCK, J.F.; WATKINS, C.B. Harvest maturity, storage temperature and relative humidity affect fruit quality, antioxidant contents and activity, and inhibition of cell proliferation of strawberry fruit. Postharvest Biology and Technology, v.49, p.201-209, 2008. DOI: 10.1016/j. postharvbio.2008.02.008.

SILVA, M.C. da; ATARASSI, M.E.; FERREIRA, M.D.; MOSCA, M.A. Qualidade pós-colheita de caqui 'Fuyu' com utilização de diferentes concentrações de cobertura comestível. Ciência Agrotécnica, v.35, p.144-151, 2011.

STRIK, B.C.; CLARK, J.R.; FINN, C.E.; BAÑADOS, M.P. Worldwide blackberry production. HortTechnology, v.17, p.205-213, 2007.

VIZZOTTO, M.; RASEIRA, M. do C.B.; PEREIRA, M.C.; FETTER, M. da R. Teor de compostos fenólicos e atividade antioxidante em diferentes genótipos de amoreira-preta (Rubus sp.). Revista Brasileira de Fruticultura, v.34, p.853-858, 2012.

WANG, C.Y.; WANG, S.Y. Effect of storage temperatures on fruit quality of various cranberry cultivars. Acta Horticulturae, v.810, p.853-862, 2009. DOI: 10.17660/ActaHortic.2009.810.114.

YANG, Y.; WANG, J.; XING, Z.; DAI, Y.; CHEN, M. Identification of phenolics in Chinese toon and analysis of their content changes during storage. Food Chemistry, v.128, p.831-838, 2011. DOI: 10.1016/j.foodchem.2011.03.071.

Recebido em 2 de março de 2016 e aprovado em 25 de maio de 2016

Pesq. agropec. bras., Brasília, v.51, n.8, p.950-957, ago. 2016 DOI: $10.1590 / \mathrm{S} 0100-204 X 2016000800007$ 